\title{
НАУЧНО-ТЕХНИЧЕСКИЙ ПРОГРЕСС И НЕЙТРАЛЬНОСТЬ ПО ХИКСУ, ХАРРОДУ И СОЛОУ: ГЕНЕЗИС, ПОСТРОЕНИЕ И ОБОБЩЕНИЯ
}

\author{
А.Ф. Проневич, Г.А. Хацкевич*
}

Анализируются понятия нейтральности научно-технического прогресса (НТП) по Хиксу, Харроду и Солоу. Описаны множества агрегированных производственных функций, учитывающих нейтральный, расширенно нейтральный и полностью нейтральный по Хиксу НТП. Указаны аналитические виды динамических производственных функций, учитывающих нейтральный по Харроду НТП и нейтральный по Солоу НТП. Выделены классы однородных производственных функций, учитывающих НТП, нейтральный по Хиксу, Харроду и Солоу. Выявлены аналитические связи между понятиями - нейтральный, расширенно нейтральный и полностью нейтральный по Хиксу НТП. Предложены обобщения понятий «нейтральность по Хиксу», «нейтральность по Харроду» и «нейтральность по Солоу», а также установлены соответствующие им классы агрегированных динамических производственных функций. Приведены аналитические критерии того, что агрегированные производственные функции учитывают НТП, нейтральный по Хиксу, Харроду и Солоу.

Ключевые слова: научно-технический прогресс, производственная функция, нейтральность по Хиксу, нейтральность по Харроду, нейтральность по Солоу.

JEL-классификация: C65, О30.

DOI: $10.46782 / 1818-4510-2020-3-87-105$

Материал поступил 2.07.2020 г.

Научно-технический прогресс (НТП) - это развитие техники и технологии производства, а также рост организации производства, повышение технического уровня кадров, изменение их профессиональной структуры и другие факторы; необходимая предпосылка расширенного воспроизводства ${ }^{1}$. Экономико-математический анализ НТП наиболее полно проводится с помощью теории производственных функций² (Плакунов, Раяцкас, 1984; Клейнер, 1986).

Рассмотрим динамическую агрегированную производственную функцию (ПФ)

$$
Y=F(K, L, t),
$$

где $\quad Y$ - выпуск продукции;

$K$ - капитал;

$L-$ труд;

$t$ - параметр времени из числового луча $\mathbf{R}_{+}=[0 ;+\infty)$, каждое значение которого выражает определенный уровень НТП, а неотрицательная функция $F$ является дважды непрерывно дифференцируемой на множестве $D=G \times \mathbf{R}_{+}$, экономическая область $G \subset \mathbf{R}_{+}^{2}=\{(K, L): K \geq 0, L \geq 0\}$.

Начиная с 20-х годов XX в. исследователи пытались понять, в чем состоит НТП с точки зрения макроэкономической динамики, какие экономические показатели он оставляет неизменными (нейтральными, инвариантными) во времени, а какие - изменяет.

1 Лопатников Л.И. 2003. Экономико-математический словарь: словарь современной экономической науки. Москва: Дело. С. 214-215.

2 Горбунов В.К. 2013. Производственные функщии: теория и построение: учебное пособие. Ульяновск: УлГУ.

* Проневич Андрей Францевич (pranevich@grsu.by), кандидат физико-математических наук, доцент, Гродненский государственный университет им. Янки Купалы (г. Гродно, Беларусь);

Хацкевич Геннадий Алексеевич (khatskevich@sbmt.by), доктор экономических наук, профессор, Институт бизнеса Белорусского государственного университета (г. Минск, Беларусь). 
Одна из первых классификаций НТП была предложена в 1920 г. профессором Кембриджского университета А.С. Пигу в работе «Экономическая теория благосостояния» ${ }^{3}$ : «изобретения или нововведения, уменьшающие отношение капитала к труду в той отрасли, где они внедряются, будут капиталосберегающими, изобретения или нововведения, увеличивающие это отношение, трудосберегающими, а изобретения или нововведения, оставляющие его неизменным, нейтраль-

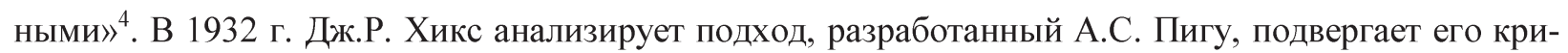
тике и предлагает свою классификацию ${ }^{5}$, основанную на изменении с течением времени предельной нормы технического замещения ${ }^{6}$ : Если рассматривать два фактора, «труд» и «капитал», то изобретения можно классифицировать в соответствии с тем, увеличивают ли они, оставляют неизменным либо уменьшают отношение предельной производительности капитала к предельной производительности труда по сравнению с ее первоначальным состоянием. Такие изобретения будем называть «трудосберегающими», «нейтральными» и «капиталосберегающими» соответственно». Дж.В. Робинсон в своей монографии «Очерки по теории занятости» ${ }^{7}$ при обсуждении влияния технологий на положение долгосрочного равновесия в теории занятости использовала классификацию профессора Дж.Р. Хикса НТП при дополнительном условии ${ }^{8}$ : «фондовооруженность труда является величиной постоянной» ${ }^{9}$. В дальнейшем данная модификация определения нейтральности НТП по Хиксу получила широкое распространение (Uzawa, 1961; Sato, 1965; Sato, Beckmann, 1968; Stiglitz, Uzawa, 1969. Р. 20; Ferguson, 1969. Р. 217; Курзенев, Матвеенко, 2018. С. 73-74) ${ }^{10}$ и сегодня в научной литературе используется в качестве основного понятия.

Идея еще одной классификации НТП была заложена Р.Ф. Харродом в рецензии ${ }^{11}$ на книгу Дж.В. Робинсон «Очерки по теории занятости» и позднее в расширенном виде изложена в его монографии «К динамической экономической теории» ${ }^{12}$. Подробному и глубокому изучению вопросов истории возникновения и становления понятия «нейтральность НТП по Харроду», а также возможности его использования в теории экономического роста (через дискуссии и переписку Р.Ф. Харрода с экономистами Н. Калдором, Р.Ф. Каном, Дж.М. Кейнсом, Дж.В. Робинсон, П. Сраффа, Р.Дж. Хоутри и др.) посвящена работа профессора Д. Бесоми (Besomi, 1999).

Понятие «нейтральность НТП по Солоу», которое является симметричным по отношению к понятию «нейтральность НТП по Харроду», было введено и использовано в работах американского экономиста P.М. Солоу (Solow, 1960; Solow, 1962; Solow, 1963).

Для неоклассических ПФ (1) в статье Р. Сато и М. Бекмана (Sato, Beckmann, 1968) в зависимости от инвариантности относительно НТП различных соотношений (рассмотрены 15 случаев) между основными экономико-математическими характеристиками ПФ введены возможные опре-

\footnotetext{
${ }^{3}$ Pigou A.C. 1920. The economics of welfare. London: Macmillan. P. 719.

${ }^{4} \ll \ldots$ an invention or improvement which reduces the ratio of capital to labour in the industry to which it applies will be capital-saving, one which increases it labour-saving, and one which leaves it unchanged, neutral».

5 «If we concentrate on two groups of factors, «labour» and «capital», and suppose them to exhaust the list, then we can classify inventions according as their initial effects are to increase, leave unchanged, or diminish the ratio of the marginal product of capital to that of labour. We may call these inventions «labour-saving», «neutral», and «capital-saving respectively».

${ }^{6}$ Hicks J.R. 1932. The theory of wages. London: Macmillan. PP. 121-122.

${ }^{7}$ Robinson J. 1937. Essays in the theory of employment. London: Macmillan.

8 «I made use of Mr. Hicks's classification of inventions, according to which an invention is said to be neutral when it raises the marginal productivities of labour and capital in the same proportion, and is said to be labour-saving or capital-saving according as it raises the marginal productivity of capital more or less than that of labour, the amounts of the factors being unchanged» (Robinson, 1938).

${ }^{9}$ Robinson J. 1938. The classification of inventions. The Review of Economic Studies. Vol. 5(2). PP. 139-142.

10 Дадаян В.С. (Ред.) 1973. Моделирование народно-хозяйственных процессов: учебное пособие. Москва: Экономика. С. 433-435.

${ }^{11}$ Harrod R.F. 1937. Review of Joan Robinson's «Essays in the theory of employment». Economic Journal. Vol. 47. PP. 326-330.

${ }^{12}$ Harrod R.F. 1948. Towards a dynamic economics. London: Macmillan. PP. 22-27.
} 
деления нейтральности НТП и получены соответствующие им аналитические представления динамических линейно-однородных ПФ. Приведенная классификация различных типов нейтральности НТП была апробирована на статистических данных США, Японии и Германии (Beckmann, Sato, 1969). Аналитические формы линейно-однородных динамических ПФ, которые одновременно учитывают различные типы нейтральности по классификации Сато - Бекмана, были выделены в работе (Gehrig, 1980).

Отметим также и то, что широкое распространение в макроэкономическом анализе получила классификация НТП, разработанная выдающимся польским экономистом М. Калецким (Osiatynski, 1993. P. 53; Chilosi, Gomulka, 1969).

Вехи становления и развития теории НТП, его влияние на экономический рост, а также обзор научной литературы по этому направлению приведены в (Браун, 1971; Варшавский, 1984; Плакунов, Раяцкас, 1984; Анчишкин, 2003; Барро, Сала-и-Мартин, 2017; Курзенев, Матвеенко, 2018) ${ }^{13}$.

Данная работа продолжает исследования ${ }^{14}$ (Хацкевич, Проневич, 2017; Хацкевич, Проневич, Чайковский, 2019; Khatskevich, Pranevich, 2017, 2018; Khatskevich, Pranevich, Karaleu, 2019) по изучению показателей эффективности процесса замещения факторов производства. В статье описаны классы агрегированных динамических ПФ, учитывающих нейтральный по Хиксу, по Харроду и по Солоу НТП. Предложены обобщения понятий «нейтральность по Хиксу», «нейтральность по Харроду» и «нейтральность по Солоу».

\section{Научно-технический прогресс и нейтральность по Хиксу}

НТП, нейтральный по Хиксу. НТП будем называть нейтральным по Хиксу ${ }^{15}$ (Курзенев, Матвеенко, 2018. С. 73-74), если предельная норма технического замещения (труда капиталом) ${ }^{16}$ $M R T S_{L K}$ не изменяется с течением времени при фиксированной фондовооруженности труда $k=K / L$, т. е. $\operatorname{MRTS}_{L K}(F)=$ const при $K / L=$ const.

Если при постоянной фондовооруженности труда предельная норма замещения возрастает со временем, т. е. $\partial_{t} M R T S_{L K}(F)>0$ при $K / L=$ const, то имеет место трудоемкий по Хиксу тип экономического развития. Если при постоянной фондовооруженности труда предельная норма заме-

${ }^{13}$ Гранберг А.Г. 1988. Моделирование соџиалистической экономики: учебник. Москва: Экономика; Дадаян В.С. (Ред.) 1973. Моделирование народно-хозяйственных проиессов: учебное пособие. Москва: Экономика; Иванилов Ю.П., Лотов А.В. 1979. Математические модели в экономике: учебное пособие. Москва: Наука.

${ }^{14}$ Проневич А.Ф., Хацкевич Г.А. 2020. Голотетичные производственные функции по линейному экзогенному научно-техническому прогрессу. Многомерный статистический анализ, эконометрика и моделирование реальных процессов: труды Х Международной школы-семинара. Москва: ЦЭМИ РАН. С. 120-122.

15 Дадаян В.С. (Ред.) 1973. Моделирование народно-хозяйственных процессов: учебное пособие. Москва: Экономика. С. 434.

${ }^{16}$ Предельная норма технического замещения (труда капиталом) $\operatorname{MRTS}_{L K}(F)=-\frac{d K}{d L}=\frac{\partial_{L} F(K, L, t)}{\partial_{K} F(K, L, t)}$ является для динамической ПФ (1) характеристикой первого порядка (относительно производных) и на языке процентов приближенно показывает, на сколько процентов нужно увеличить или уменьшить использование капитала $K$ при уменьшении или увеличении труда $L$ на $1 \%$ для того, чтобы сохранить выпуск продукции $Y$ неизменным при заданном уровне НТП $t$ (Тарасевич Л.С., Гребенников П.И., Леусский А.И. 2015. Микроэкономика: учебник и практикум. Москва: Юрайт. С. 4142). Графически характеристика MRTS представляется тангенсом угла наклона касательной к изокванте ПФ в точке, указывающей необходимые объемы труда и капитала для производства заданного объема продукции. Предельная норма технического замещения (капитала трудом) $\operatorname{MRTS}_{K L}(F)=1 / \operatorname{MRTS}_{L K}(F)$. Здесь и далее для удобства вычислений через $\partial_{t}, \partial_{K}$ и $\partial_{L}$ обозначены частные производные первого порядка по переменным $t, K$ и $L$ соответственно, а через $\partial_{K t}^{2}, \partial_{L t}^{2}-$ смешанные частные производные второго порядка по соответствующим переменным. 
щения убывает с течением времени, т. е. $\partial_{t} \operatorname{MRTS}_{L K}(F)<0$ при $K / L=$ const, то имеет место капиталоемкий (или фондоемкий) по Хиксу тип развития.

При нейтральном по Хиксу НТП функция $M R T S_{L K}$ не зависит от параметра НТП $t$, а является некоторой функцией ${ }^{17} h$ от фондовооруженности труда:

$$
\frac{\partial_{L} F(K, L, t)}{\partial_{K} F(K, L, t)}=h\left(\frac{K}{L}\right)
$$

Множество функций, учитывающих нейтральный по Хиксу НТП, обозначим через $H N$. Например, динамическая ПФ Викселля - Тинбергена ${ }^{18}$

$$
F(K, L, t)=A K^{\alpha} L^{\beta} e^{\gamma t}, A>0, \alpha, \beta, \gamma \in \mathbf{R} \backslash\{0\}
$$

учитывает НТП, нейтральный по Хиксу, ибо предельная норма технического замещения (труда капиталом) $\operatorname{MRTS}_{L K}(F)=\frac{\beta}{\alpha} \frac{K}{L}$ является постоянной величиной при $K / L=$ const.

Опишем понятие нейтральности НТП по Хиксу в терминах непрерывных темпов прироста ${ }^{19}$ предельных производительностей труда и капитала. Для этого представим частную производную по времени от предельной нормы технического замещения (труда капиталом) в следующем виде ${ }^{20}$ :

$$
\begin{aligned}
& \quad \partial_{t} \operatorname{MRTS}_{L K}(F)=\partial_{t}\left(\frac{\partial_{L} F}{\partial_{K} F}\right)=\frac{\partial_{t}\left(\partial_{L} F\right) \cdot \partial_{K} F-\partial_{L} F \cdot \partial_{t}\left(\partial_{K} F\right)}{\left(\partial_{K} F\right)^{2}}=\frac{\partial_{t}\left(\partial_{L} F\right)}{\partial_{K} F}-\frac{\partial_{t}\left(\partial_{K} F\right)}{\partial_{K} F} \cdot \frac{\partial_{L} F}{\partial_{K} F}= \\
& =\frac{\partial_{t}\left(\partial_{L} F\right)}{\partial_{L} F} \cdot \frac{\partial_{L} F}{\partial_{K} F}-\frac{\partial_{t}\left(\partial_{K} F\right)}{\partial_{K} F} \cdot \frac{\partial_{L} F}{\partial_{K} F}=\left(\partial_{t}\left(\ln \partial_{L} F\right)-\partial_{t}\left(\ln \partial_{K} F\right)\right) \frac{\partial_{L} F}{\partial_{K} F}=\left(T_{L}-T_{K}\right) M R T S_{L K}(F),
\end{aligned}
$$

где $T_{K}=\partial_{t}\left(\ln \partial_{K} F\right)$ и $T_{L}=\partial_{t}\left(\ln \partial_{L} F\right)$ - темпы прироста предельной производительности капитала и труда соответственно.

Основываясь на соотношениях темпов приростов предельных производительностей труда и капитала, получаем: 1) если темп прироста предельной производительности труда $T_{L}$ превышает темп прироста предельной производительности капитала $T_{K}$, т. е. $T_{L}>T_{K}$, то имеет место трудоемкий (по Хиксу) НТП; 2) если темп прироста предельной производительности труда $T_{L}$ равен темпу прироста предельной производительности капитала $T_{K}$, т. е. $T_{L}=T_{K}$, а значит, рост вовлечения в производство дополнительного числа занятых равен росту привлечения в производство капитала, то имеет место НТП, нейтральный по Хиксу; 3) если темп прироста предельной производительности труда $T_{L}$ меньше темпа прироста предельной производительности капитала $T_{K}$, т. е. $T_{L}<T_{K}$, то имеет место капиталоемкий (по Хиксу) НТП.

Класс агрегированных ПФ типа $H N$ описывается следующими утверждениями.

\footnotetext{
17 Функция $h$ будет непрерывно дифференцируемой, так как при задании динамической агрегированной ПФ (1) положено, что функция $F$ является дважды непрерывно дифференцируемой.

18 Tinbergen J. 1942. Professor Douglas' Production Function. Review of the International Statistical Institute. Vol. 10. No $1 / 2$. PP. $37-48$.

19 Гранберг А.Г. 1988. Моделирование соииалистической экономики: учебник. Москва: Экономика. С. $256-257$.

20 Дадаян В.С. (Ред.) 1973. Моделирование народно-хозяйственных прочессов: учебное пособие. Москва: Экономика. С. 434.
} 
$\mathbf{1}^{\circ}$. Динамическая ПФ (1) учитывает нейтральный по Хиксу НТП тогда и только тогда, когда ее можно представить в аналитическом виде

$$
F(K, L, t)=\Phi(\Psi(K, L), t),
$$

где $\Phi$ - некоторая неотрицательная непрерывно дифференцируемая функция переменных $\Psi$ и $t$, а $\Psi$ - линейно-однородная непрерывно дифференцируемая функция от $K$ и $L$.

Так, например, по утверждению $1^{\circ}$, ПФ Викселля - Тинбергена (3) учитывает нейтральный по Хиксу НТП, ибо ее можно представить в форме (4) с внешней функцией $\Phi(\Psi, t)=A \Psi^{\alpha+\beta} e^{\gamma t}$ и внутренней линейно-однородной функцией $\Psi(K, L)=K^{\alpha /(\alpha+\beta)} L^{\beta /(\alpha+\beta)}$.

$\mathbf{2}^{\circ}$. Однородная степени $q \in \mathbf{R} \backslash\{0\}$ ПФ (1) учитывает нейтральный по Хиксу НТП, если и только если она может быть представлена в аналитической форме

$$
F(K, L, t)=A(t) \Theta(K, L),
$$

где $\Theta$ - некоторая неотрицательная однородная степени $q$ непрерывно дифференцируемая функция, а строго возрастающая функция $A$ такая, что $A(0)=1$, есть индекс НТП.

НТП, расширенно нейтральный по Хиксу. НТП назовем расширенно нейтральнылм по Хиксу (Hicks, 1932. Р. 121-122; Morimoto, 1974; Blackorby, Lovell, Thursby, 1976), если предельная норма замещения (труда капиталом) $\operatorname{MRTS}_{L K}(F)$ не зависит от параметра НТП $t$.

Таким образом, динамическая ПФ (1) учитывает НТП, расширенно нейтральный по Хиксу, если и только если имеет место тождество

$$
\frac{\partial_{L} F(K, L, t)}{\partial_{K} F(K, L, t)}=h(K, L)
$$

Множество ПФ, учитывающих расширенно нейтральный по Хиксу НТП, обозначим через $E H N$. Отметим, что множество $H N \subset E H N$. Для ПФ типа $E H N$ верны следующие утверждения.

$3^{\circ}$. (Критерий НТП, расширенно нейтрального по Хиксу.) ПФ (1) учитывает НТП, расширенно нейтральный по Хиксу, если и только если верно тождество $\partial_{t} M R T S_{L K}(F)=0$.

$4^{\circ}$. (Leontief, 1947; Sono, 1961; Morimoto, 1974) ПФ (1) учитывает расширенно нейтральный по Хиксу НТП тогда и только тогда, когда ее можно представить в виде

$$
F(K, L, t)=\Phi(\Psi(K, L), t),
$$

где $\Phi$ - некоторая неотрицательная непрерывно дифференцируемая функция двух переменных $\Psi$ и $t$, а $\Psi$ - непрерывно дифференцируемая функция на области $G$ от $K$ и $L$.

$\mathbf{5}^{\circ}$. Однородная степени $q \in \mathbf{R} \backslash\{0\}$ ПФ (1) учитывает расширенно нейтральный по Хиксу НТП в том и только в том случае, когда она может быть представлена в виде (5).

Основываясь на утверждениях $2^{\circ}$ и $5^{\circ}$, получаем следующую закономерность.

$\mathbf{6}^{\circ}$. Однородная степени $q \in \mathbf{R} \backslash\{0\}$ ПФ (1) учитывает нейтральный по Хиксу НТП тогда и только тогда, когда она учитывает расширенно нейтральный по Хиксу НТП.

НТП, польностью нейтральный по Хиксу. Будем говорить, что НТП является полностью нейтральным по Хиксу (Blackorby, Lovell, Thursby, 1976), если для динамической ПФ (1), учитывающей этот НТП, выполняется система тождеств

$$
\partial_{K}(\ln F(K, L, t))=\varphi(K, L), \partial_{L}(\ln F(K, L, t))=\psi(K, L),
$$


где $\varphi$ и $\psi$ - некоторые непрерывно дифференцируемые на экономической области $G$ функции, которые не зависят от параметра НТП $t$.

Множество ПФ, учитывающих полностью нейтральный по Хиксу НТП, обозначим через $C H N$. Отметим, что $C H N \subset E H N$, ибо в силу тождеств (7) имеем

$$
\operatorname{MRTS}_{L K}(F)=\frac{\partial_{L} F(K, L, t)}{\partial_{K} F(K, L, t)}=\frac{\partial_{L} F(K, L, t)}{F(K, L, t)} \cdot \frac{F(K, L, t)}{\partial_{K} F(K, L, t)}=\frac{\partial_{L} \ln F(K, L, t)}{\partial_{K} \ln F(K, L, t)}=\frac{\varphi(K, L)}{\psi(K, L)},
$$

а значит, верно тождество (6) при $h(K, L)=\varphi(K, L) / \psi(K, L)$.

Общий вид ПФ, учитывающих полностью нейтральный по Хиксу НТП, описывает следующее утверждение.

$7^{\circ}$. (Blackorby, Lovell, Thursby, 1976) Динамическая ПФ (1) учитывает НТП, полностью нейтральный по Хиксу, если и только если ее можно представить в виде

$$
F(K, L, t)=A(t) \Lambda(K, L),
$$

где $\Lambda$ - некоторая неотрицательная непрерывно дифференцируемая функция на области $G$, а строго возрастающая функция $A$ такая, что $A(0)=1$, есть индекс НТП.

На основании утверждения $7^{\circ}$ и понятия «продуктоувеличивающий НТП» ${ }^{21}$ получаем следующую закономерность.

$\mathbf{8}^{\circ}$. НТП является полностью нейтральным по Хиксу в том и только в том случае, когда он является продуктоувеличивающим. Следовательно, понятия «продуктоувеличивающий НТП» и «полностью нейтральный по Хиксу НТП» равносильны и их можно использовать на равных правах (как синонимы).

$9^{\circ}$. (Критерий полной нейтральности по Хиксу НТП.) Для того, чтобы ПФ (1) учитывала НТП, полностью нейтральный по Хиксу, необходимо и достаточно выполнения тождества

$$
\partial_{t} \ln F(K, L, t)=\theta(t)
$$

где $\theta$ - некоторая функция (темп прироста индекса НТП), которая зависит только от параметра НТП.

Исходя из тождества (9) рассмотрим следующие возможности:

Случай 1. Пусть $\theta(t)=0 \quad \forall t \in T$, т. е. имеет место нулевой темп прироста индекса НТП. Тогда на основании тождества (9) получаем, что $A(t)=a=$ const и ПФ $F(K, L, t)=a \Lambda(K, L)$ является стационарной, т. е. не зависит от параметра НТП $t$.

Случай 2. Пусть $\theta(t)=\lambda \quad \forall t \in T, \lambda \in \mathbf{R} \backslash\{0\}$, т. е. имеет место постоянный темп прироста индекса НТП. Тогда на основании тождества (9) получаем, что $A^{\prime}(t) / A(t)=\lambda$, а значит, индекс НТП $A(t)=a e^{\lambda t} \forall t \in T$, число $a>0$, и ПФ $F(K, L, t)=a e^{\lambda t} \Lambda(K, L)$ принимает аналитическую форму Тинбергена.

Случай 3. В общем случае, когда темп прироста индекса НТП задан в виде непрерывной функции $\theta(t)$, на основании тождества (9) получаем, что $A^{\prime}(t) / A(t)=\theta(t)$, а значит, индекс НТП $A(t)=a \exp \int \theta(t) d t$, число $a>0$, и ПФ $F(K, L, t)=a \Lambda(K, L) \exp \int \theta(t) d t$.

21 Черемных Ю.Н. 2008. Микроэкономика. Продвинутый уровень: учебник. Москва: ИНФРА-М. С. 224; Иванилов Ю.П., Лотов А.В. 1979. Математические модели в экономике: учебное пособие. Москва: Наука. С. 83-85. 
Класс ПФ, учитывающих как нейтральный по Хиксу НТП, так и полностью нейтральный по Хиксу НТП, описывает следующее утверждение.

$\mathbf{1 0}^{\circ}$. Динамическая ПФ (1), учитывающая полностью нейтральный по Хиксу НТП, будет также учитывать и нейтральный по Хиксу НТП, если и только если ее можно представить в аналитической форме

$$
F(K, L, t)=A(t) H(\Psi(K, L))
$$

где $H$ - некоторая неотрицательная непрерывно дифференцируемая функция, $\Psi$ - линейнооднородная непрерывно дифференцируемая функция двух переменных $K$ и $L$, а строго возрастающая функция $A$ такая, что $A(0)=1$, есть индекс НТП.

НТП, обобщенно нейтральный по Хиксу. НТП назовем обобщенно нейтральным по Хиксу, если предельная норма технического замещения (труда капиталом) $M R T S_{L K}$ не изменяется с течением времени вдоль траекторий развития экономики ${ }^{22} \psi(K, L)=C$, т. е.

$$
\operatorname{MRTS}_{L K}(F)=\text { const при } \psi(K, L)=C \text {. }
$$

Таким образом, при обобщенно нейтральном по Хиксу НТП функция $M R T S_{L K}$ не зависит от параметра НТП $t$, а является некоторой непрерывно дифференцируемой функцией $h$ от функции $\psi$, задающей траектории развития экономики, т. е.

$$
\frac{\partial_{L} F(K, L, t)}{\partial_{K} F(K, L, t)}=h(\psi(K, L)) .
$$

Множество динамических ПФ, учитывающих обобщенно нейтральный по Хиксу НТП, обозначим через $G H N$. Отметим очевидное включение $H N \subset G H N \subset E H N$.

В качестве иллюстрации определения (11) опишем обобщенно нейтральный по Хиксу НТП вдоль траекторий развития экономики, заданных линейными функциями

$$
a K+b L=C, a \in \mathbf{R} \backslash\{0\}, b \in \mathbf{R} .
$$

11․ Динамическая ПФ (1) учитывает обобщенно нейтральный по Хиксу НТП вдоль линейных траекторий развития экономики (13) тогда и только тогда, когда ее можно представить в аналитическом виде

$$
F(K, L, t)=\Phi(L+\Psi(a K+b L), t)
$$

где $\Phi$ и $\Psi$ - некоторые непрерывно дифференцируемые функции, причем функция $\Phi$ неотрицательная.

$\mathbf{1 2}^{\circ}$. Однородная степени $q \in \mathbf{R} \backslash\{0\}$ ПФ (1) учитывает обобщенно нейтральный по Хиксу НТП вдоль линейных траекторий развития экономики (13), если и только если она может быть представлена в аналитической форме

22 Траектории развития экономики (Гамецкий, Соломон, 1997) заданы в неявной форме в виде семейства кривых $\psi(K, L)=C$, лежащих в первом квадранте координатной плоскости $O K L$, где $\psi-$ непрерывно дифференцируемая функция на экономической $G$, а $C$ - произвольная вещественная постоянная. Например, для нейтрального по Хиксу НТП траектории развития экономики $K / L=C$-лучи, выходящие из начала координат и расположенные в первом квадранте $\mathbf{R}_{+}^{2}$ координатной плоскости $O K L$. 


$$
F=A(t)\left(a_{1} K+b_{1} L\right)^{q},
$$

где строго возрастающая функция $A$ такая, что $A(0)=1$, есть индекс НТП, а $a_{1}$ и $b_{1}$ - некоторые вещественные числа.

\section{Научно-технический прогресс и нейтральность по Харроду}

НТП, нейтральный по Харроду. НТП является нейтральным по Харроду (Барро, Сала-иМартин, 2018. С. 73) ${ }^{23}$, если предельная производительность капитала ${ }^{24} M P_{K}$ не изменяется с течением времени при фиксированной фондоотдаче, т. е.

$$
M P_{K}(F)=\text { const при } Y / K=\text { const. }
$$

Следовательно, при нейтральном по Харроду НТП функция $M P_{K}$ не зависит явно от параметра НТП $t$, а является некоторой непрерывно дифференцируемой функцией $h$ от фондоотдачи (производительности капитала), т. е. $\partial_{K} F(K, L, t)=h\left(\frac{Y}{K}\right)$.

Например, динамическая ПФ Викселля - Тинбергена (3) учитывает НТП, нейтральный по Харроду, ибо предельная производительность капитала

$$
M P_{K}(F)=\partial_{K}\left(A K^{\alpha} L^{\beta} e^{\gamma t}\right)=\alpha A K^{\alpha-1} L^{\beta} e^{\gamma t}=\alpha \frac{F(K, L, t)}{K}=\alpha \frac{Y}{K}
$$

является постоянной величиной при $Y / K=$ const.

Если при постоянной фондоотдаче предельная производительность капитала растет с течением времени, т. е. $\partial_{t} M P_{K}(F)>0$ при $Y / K=$ const, то имеет место капиталоемкий (или фондоемкий) по Харроду тип экономического развития. Если при постоянной фондоотдаче предельная производительность капитала убывает с течением времени, т. е. $\partial_{t} M P_{K}(F)<0$ при $Y / K=$ const, то будет трудоемкий по Харроду тип экономического развития.

$\mathbf{1 3}^{\circ}$. (Morimoto, 1974) Динамическая ПФ (1) учитывает нейтральный по Харроду НТП тогда и только тогда, когда ее можно представить в аналитическом виде

$$
F(K, L, t)=\Phi(K, \Psi(L, t))
$$

где $Ф$ - некоторая неотрицательная линейно-однородная непрерывно дифференцируемая функция двух переменных $K$ и $\Psi$, а $\Psi$ - непрерывно дифференцируемая функция от $L$ и $t$.

Так, например, однородную степени $\alpha+\beta$ ПФ Викселля - Тинбергена (3), учитывающую нейтральный по Харроду НТП, по утверждению $13^{\circ}$, можно представить в виде сложной функции вида (16) с внешней линейно-однородной функцией $\Phi(K, \Psi)=A K^{\alpha} \Psi^{1-\alpha}$ и внутренней функцией $\Psi(L, t)=L^{\beta /(1-\alpha)} e^{(\gamma /(1-\alpha)) t}$.

23 Дадаян В.С. (Ред.) 1973. Моделирование народно-хозяйственных процессов: учебное пособие. Москва: Экономика. С. 435.

${ }^{24}$ Предельная производительность капитала (труда) $M P_{K}(F)=\partial_{K} F(K, L, t) \quad\left(M P_{L}(F)=\partial_{L} F(K, L, t)\right)$ приближенно показывает, на сколько изменится объем выпуска продукции в случае использования дополнительной единицы капитала (труда) и при неизменном количестве труда (капитала) и заданном уровне НТП (Альсевич В.В. 1998. Мaтематическая экономика. Конструктивная теория: учебное пособие. Минск: Дизайн ПРО. С. 70). 
14․ (Robinson, 1938; Uzawa, 1961) Линейно-однородная ПФ (1) учитывает нейтральный по Харроду НТП, если и только если она может быть представлена в аналитической форме

$$
F(K, L, t)=\Phi(K, B(t) L)
$$

где $\Phi$ - некоторая неотрицательная линейно-однородная непрерывно дифференцируемая функция, а строго возрастающая функция $B$ такая, что $B(0)=1$, есть индекс НТП по труду.

Например, ПФ Кобба - Дугласа - Тинбергена $F(K, L, t)=A K^{\alpha} L^{1-\alpha} e^{\gamma t}, A>0, \alpha \in(0 ; 1)$ является линейно-однородной и, по утверждению $14^{\circ}$, ее можно представить в виде функции $F(K, L, t)=A K^{\alpha}\left(e^{(\gamma /(1-\alpha)) t} L\right)^{1-\alpha}$, где внешняя линейно-однородная функция $\Phi(K, \Psi)=A K^{\alpha} \Psi^{1-\alpha}$ и внутренняя функция $\Psi(L, t)=B(t) L$ с индексом НТП по труду $B(t)=e^{(\gamma /(1-\alpha)) t}$.

Необходимое условие того, что динамическая ПФ (1) учитывает НТП, нейтральный по Харроду, выражает следующее утверждение.

$\mathbf{1 5}^{\circ}$. Если ПФ (1) учитывает нейтральный по Харроду НТП, то верно тождество

$$
\frac{\partial_{t} F(K, L, t)}{\partial_{L} F(K, L, t)}=\Theta(L, t)
$$

где $\Theta$ есть функция, зависящая только от переменных $L$ и $t$.

16². (Okuguchi, 1968) Динамическая линейно-однородная ПФ (1) учитывает НТП, нейтральный по Харроду, в том и только в том случае, когда имеет место тождество

$$
\frac{\partial_{t} F(K, L, t)}{L \cdot \partial_{L} F(K, L, t)}=\beta(t)
$$

где $\beta$ есть функция, зависящая только от параметра НТП $t$.

$17^{\circ}$. Однородная степени $q \in \mathbf{R} \backslash\{0\}$ ПФ (1) одновременно учитывает как нейтральный по Хиксу НТП, так и нейтральный по Харроду НТП, если и только если она может быть представлена в аналитической форме Викселля - Тинбергена:

$$
F(K, L, t)=A(t) K^{\alpha} L^{\beta}, \alpha \in \mathbf{R} \backslash\{0 ; 1\}, \beta \in \mathbf{R} \backslash\{0\}, \alpha+\beta=q,
$$

где строго возрастающая функция $A$ есть индекс НТП.

Основываясь на утверждениях $2^{\circ}, 6^{\circ}, 7^{\circ}$ и $17^{\circ}$, получаем следующую закономерность.

$\mathbf{1 8}^{\circ}$. Пусть ПФ (1) является однородной степени $q \in \mathbf{R} \backslash\{0\}$. Тогда она будет одновременно учитывать как нейтральный (расширенно нейтральный или полностью нейтральный) по Хиксу НТП, так и нейтральный по Харроду НТП тогда и только тогда, когда она может быть представлена в аналитической форме Викселля - Тинбергена (18).

НТП, обобщенно нейтральный по Харроду. НТП назовем обобщенно нейтральным по Харроду, если предельная производительность капитала не изменяется с течением времени при фиксированной средней отдаче обобщенного ресурса ${ }^{25}$, т. е.

$$
M P_{K}(F)=\text { const при } \frac{Y}{\alpha K+\beta L}=\text { const }(\alpha>0, \beta \geq 0) .
$$

${ }^{25}$ В качестве такого обобщенного ресурса чаще всего рассматривается себестоимость продукции. 
Отметим, что из определения обобщенной нейтральности НТП по Харроду (19) при $\beta=0$ следует определение НТП, нейтрального по Харроду.

Таким образом, при обобщенно нейтральном по Харроду НТП функция $M P_{K}$ не зависит явно от параметра НТП, а является некоторой непрерывно дифференцируемой функцией $h$ от средней отдачи обобщенного ресурса, т. е.

$$
\partial_{K} F(K, L, t)=h\left(\frac{Y}{\alpha K+\beta L}\right) .
$$

Класс ПФ, учитывающих обобщенно нейтральный по Харроду НТП, описывает следующее выражение.

$\mathbf{1 9}^{\circ}$. Динамическая ПФ (1) учитывает обобщенно нейтральный по Харроду НТП тогда и только тогда, когда ее можно представить в аналитическом виде

$$
F(K, L, t)=\Phi(\alpha K+\beta L, \Psi(L, t)),
$$

где $\Phi$ - некоторая неотрицательная линейно-однородная непрерывно дифференцируемая функция двух переменных, а $\Psi$ - непрерывно дифференцируемая функция от $L$ и $t$.

$\mathbf{2 0}^{\circ}$. Линейно-однородная ПФ (1) учитывает обобщенно нейтральный по Харроду НТП, если и только если она может быть представлена в аналитической форме

$$
F(K, L, t)=\Phi(\alpha K+\beta L, B(t) L)
$$

где $\Phi$ - некоторая неотрицательная линейно-однородная непрерывно дифференцируемая функция, а строго возрастающая функция $B$ такая, что $B(0)=1$, есть индекс НТП по труду.

\section{Научно-технический прогресс и нейтральность по Солоу}

НТП, нейтральный по Солоу. НТП является нейтральным по Солоу (Барро, Сала-иМартин, 2018. С. 73-74) ${ }^{26}$, если предельная производительность труда $M P_{L}$ не изменяется с течением времени при фиксированной производительности труда, т. е.

$$
M P_{L}(F)=\text { const при } Y / L=\text { const. }
$$

Следовательно, по определению (22), при нейтральном по Солоу НТП функция $M P_{L}$ не зависит явно от параметра НТП $t$, а является некоторой непрерывно дифференцируемой функцией $h$ от производительности труда, т. е. $\partial_{L} F(K, L, t)=h\left(\frac{Y}{L}\right)$.

Например, ПФ Викселля - Тинбергена (3) учитывает НТП, нейтральный по Солоу, ибо предельная производительность труда $M P_{L}(F)=\partial_{L}\left(A K^{\alpha} L^{\beta} e^{\gamma t}\right)=\beta \frac{F(K, L, t)}{L}=\beta \frac{Y}{L}$ является постоянной величиной при $Y / L=$ const.

Если при постоянной производительности труда предельная производительность труда растет с течением времени, т. е. $\partial_{t} M P_{L}(F)>0$ при $Y / L=$ const, то имеет место трудоемкий по Солоу тип экономического развития. Если при постоянной производительности труда предельная произ-

${ }^{26}$ Ашманов С.А. 1984. Введение в математическую экономику: учебное пособие. Москва: Наука. С. 235. 
водительность труда убывает с течением времени, т. е. $\partial_{t} M P_{L}(F)<0$ при $Y / L=$ const, то будет капиталоемкий (фондоемкий) по Солоу тип экономического развития.

Отметим, что нейтральность НТП по Солоу является симметричной по отношению к нейтральности НТП по Харроду (утверждения 21-26 доказываются методами аналогично использованным при доказательстве соответствующих утверждений 13-18).

$\mathbf{2 1}^{\circ}$. Динамическая ПФ (1) учитывает нейтральный по Солоу НТП тогда и только тогда, когда ее можно представить в аналитическом виде

$$
F(K, L, t)=\Phi(\Psi(K, t), L),
$$

где $\Phi$ - некоторая неотрицательная линейно-однородная непрерывно дифференцируемая функция двух переменных $\Psi$ и $L$, а $\Psi$ - непрерывно дифференцируемая функция от $K$ и $t$.

$\mathbf{2 2}^{\circ}$. Линейно-однородная ПФ (1) учитывает нейтральный по Солоу НТП, если и только если она может быть представлена в аналитической форме

$$
F(K, L, t)=\Phi(A(t) K, L)
$$

где $\Phi$ - неотрицательная линейно-однородная непрерывно дифференцируемая функция, а строго возрастающая функция $A$ такая, что $A(0)=1$, есть индекс НТП по капиталу.

$\mathbf{2 3}^{\circ}$. Если ПФ (1) учитывает нейтральный по Солоу НТП, то верно тождество

$$
\frac{\partial_{t} F(K, L, t)}{\partial_{K} F(K, L, t)}=\Theta(K, t)
$$

где $\Theta$ есть функция, зависящая только от переменных $K$ и $t$.

$\mathbf{2 4}^{\circ}$. (Okuguchi, 1968) Динамическая линейно-однородная ПФ (1) учитывает НТП, нейтральный по Солоу, в том и только в том случае, когда имеет место тождество

$$
\frac{\partial_{t} F(K, L, t)}{K \cdot \partial_{K} F(K, L, t)}=\alpha(t)
$$

где $\alpha$ есть функция, зависящая только от параметра НТП $t$.

$\mathbf{2 5}^{\circ}$. Однородная степени $q \in \mathbf{R} \backslash\{0\}$ ПФ (1) одновременно учитывает как нейтральный по Хиксу НТП, так и нейтральный по Солоу НТП, если и только если она может быть представлена в аналитической форме Викселля-Тинбергена (18).

$\mathbf{2 6}^{\circ}$. Пусть ПФ (1) является однородной степени $q \in \mathbf{R} \backslash\{0\}$. Тогда она будет одновременно учитывать как нейтральный (расширенно нейтральный или полностью нейтральный) по Хиксу НТП, так и нейтральный по Солоу НТП, если и только если она может быть представлена в аналитической форме Викселля - Тинбергена (18).

На основании утверждений $18^{\circ}$ и $26^{\circ}$ получаем следующую закономерность.

$27^{\circ}$. Пусть ПФ (1) является однородной степени $q \in \mathbf{R} \backslash\{0\}$. Тогда она будет одновременно учитывать нейтральный (расширенно нейтральный или полностью нейтральный) по Хиксу НТП, нейтральный по Харроду НТП и нейтральный по Солоу НТП, если и только если она может быть представлена в аналитической форме Викселля - Тинбергена (18).

НТП, обобщенно нейтральный по Солоу. НТП назовем обобщенно нейтральным по Солоу, если предельная производительность труда не изменяется с течением времени при фиксированной средней отдаче обобщенного ресурса, т. е. 


$$
M P_{L}(F)=\text { const при } \frac{Y}{\alpha K+\beta L}=\text { const }(\alpha \geq 0, \beta>0) \text {. }
$$

Отметим, что из определения обобщенной нейтральности НТП по Солоу (23) при $\alpha=0$ следует определение НТП, нейтрального по Солоу. Таким образом, при обобщенно нейтральном по Солоу НТП функция $M P_{L}$ не зависит явно от параметра НТП, а является некоторой функцией $h$ от средней отдачи обобщенного ресурса, т. е.

$$
\partial_{L} F(K, L, t)=h\left(\frac{Y}{\alpha K+\beta L}\right) .
$$

Класс ПФ, учитывающих обобщенно нейтральный по Солоу НТП, описывает следующее утверждение.

$\mathbf{2 8}^{\circ}$. Динамическая ПФ (1) учитывает обобщенно нейтральный по Солоу НТП тогда и только тогда, когда ее можно представить в аналитическом виде

$$
F(K, L, t)=\Phi(\alpha K+\beta L, \Psi(K, t)),
$$

где $\Phi$ - некоторая неотрицательная линейно-однородная непрерывно дифференцируемая функция двух переменных, а $\Psi$ - непрерывно дифференцируемая функция от $K$ и $t$.

$\mathbf{2 9}^{\circ}$. Линейно-однородная ПФ (1) учитывает обобщенно нейтральный по Солоу НТП, если и только если она может быть представлена в аналитической форме

$$
F(K, L, t)=\Phi(\alpha K+\beta L, A(t) K),
$$

где $\Phi$ - неотрицательная линейно-однородная непрерывно дифференцируемая функция, а строго возрастающая функция $A$ такая, что $A(0)=1$, есть индекс НТП по капиталу.

\section{Доказательство утверждений}

Утверждение $1^{\circ}$ - с учетом того, что функция $h(K, L)=h(K / L),-$ следует из более общего утверждения 4을 доказанного в работах (Leontief, 1947; Sono, 1961; Morimoto, 1974). Аналитический вид ПФ (4), учитывающей нейтральный по Хиксу НТП, а также формы ПФ, учитывающие нейтральный по Харроду НТП (утверждение $13^{\circ}$ ) и нейтральный по Солоу НТП (утверждение $\left.21^{\circ}\right)^{27}$

Утверждение $2^{\circ}$. По утверждению $1^{\circ}$, ПФ (1) учитывает нейтральный по Хиксу НТП, если и только если ее можно представить в виде (4). Из однородности степени $q$ функции (1) следует

$$
F(\lambda K, \lambda L, t)=\lambda^{q} F(K, L, t), \quad \forall \lambda \in(0 ;+\infty) .
$$

На основании аналитического представления (4) получаем, что левая часть

$$
F(\lambda K, \lambda L, t)=\Phi(\Psi(\lambda K, \lambda L), t)=\Phi(\lambda \Psi(K, L), t), \quad \forall \lambda \in(0 ;+\infty),
$$

правая часть $\lambda^{q} F(K, L, t)=\lambda^{q} \Phi(\Psi(K, L), t), \quad \forall \lambda \in(0 ;+\infty)$, а значит, функция $Ф$ является однородной степени $q$ по переменной $\Psi$. Поэтому верно аналитическое представление

$$
F(K, L, t)=\Phi(\Psi(K, L), t)=(\Psi(K, L))^{q} \Phi(1, t)=A(t) \Theta(K, L)
$$
C. $330-331$.

27 Прасолов А.В. 2015. Математические методы экономической динамики: учебное пособие. СПб.: Лань. 
где положено, что функция $\Theta(K, L)=(\Psi(K, L))^{q}$ и $A(t)=\Phi(1, t)$. Таким образом, имеет место представление (5). Отметим, что случай $q=1$ рассмотрен, например, в работе (Morimoto, 1974).

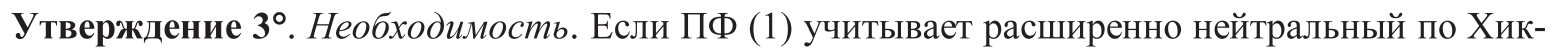
су НТП, то, используя тождество (6), находим частную производную

$$
\partial_{t} \operatorname{MRTS}_{L K}(F)=\partial_{t} \frac{\partial_{L} F(K, L, t)}{\partial_{K} F(K, L, t)}=\partial_{t} h(K, L)=0
$$

Достаточность. Решая дифференциальное уравнение в частных производных $\partial_{t} \operatorname{MRTS}_{L K}(F)=0$, получаем, что $\operatorname{MRTS}_{L K}(F)=h(K, L)$, где $h-$ произвольная функция, а значит, имеет место тождество (6).

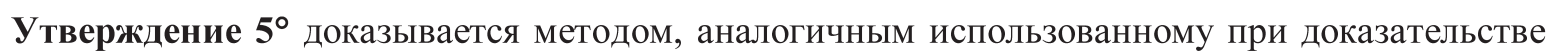
утверждения $2^{\circ}$. При этом считаем, что внешняя функция $\Phi$ является однородной степени $q_{1}$ по переменной $\Psi$, а внутренняя функция $\Psi$ является однородной степени $q_{2}$ по переменным $K$ и $L$, где числа $q_{1}, q_{2} \in \mathbf{R} \backslash\{0\}$ такие, что $q_{1}+q_{2}=q$.

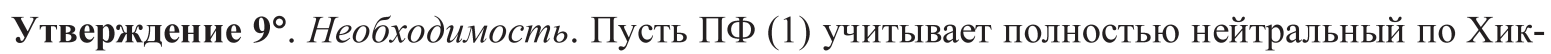
су НТП. Тогда ее можно представить в форме (8). Частная производная

$$
\partial_{t} \ln F(K, L, t)=\frac{\partial_{t} F(K, L, t)}{F(K, L, t)}=\frac{\partial_{t}(A(t) \Lambda(K, L))}{A(t) \Lambda(K, L)}=\frac{A^{\prime}(t)}{A(t)},
$$

а значит, верно тождество (9) при $\theta(t)=A^{\prime}(t) / A(t)$.

Достаточность. Пусть для ПФ (1) выполняется тождество (9). Тогда из (9) получаем

$$
\ln F(K, L, t)=\int \theta(t) d t+\tilde{\Lambda}(K, L),
$$

где $\tilde{\Lambda}$ - произвольная функция (постоянная интегрирования). Значит,

$$
F(K, L, t)=\exp \left(\int \theta(t) d t+\tilde{\Lambda}(K, L)\right)=A(t) \Lambda(K, L),
$$

где положено $A(t)=\exp \int \theta(t) d t$ и $\Lambda(K, L)=\exp \tilde{\Lambda}(K, L)$.

Таким образом, для динамической ПФ (1) верно аналитическое представление (8) и, по утверждению $7^{\circ}$, ПФ (1) учитывает полностью нейтральный по Хиксу НТП.

Утверждение $10^{\circ}$. На основании утверждения $7^{\circ}$ ПФ (1) учитывает полностью нейтральный по Хиксу НТП, если и только если она может быть представлена в форме (8). Для того чтобы она учитывала нейтральный по Хиксу НТП, необходимо и достаточно, чтобы при некоторой непрерывно дифференцируемой функции $h$ выполнялось тождество (2). Тогда из тождества

$$
\frac{\partial_{L}(A(t) \Lambda(K, L))}{\partial_{K}(A(t) \Lambda(K, L))}=h\left(\frac{K}{L}\right)
$$

получаем, что функция $\Lambda$ должна удовлетворять уравнению в частных производных

$$
\partial_{L} \Lambda-h\left(\frac{K}{L}\right) \partial_{K} \Lambda=0
$$


Из характеристического уравнения $\frac{d K}{d L}=-h\left(\frac{K}{L}\right)$, введя новую переменную $\xi=K / L$ и разделяя переменные, получим обыкновенное дифференциальное уравнение $\frac{d L}{L}=-\frac{d \xi}{h(\xi)+\xi}$. Отсюда $\ln L+\int \frac{d \xi}{h(\xi)+\xi}=\tilde{C}_{1}$, или $L \psi(\xi)=C_{1}$, где положено, что функция $\psi(\xi)=\exp \int \frac{d \xi}{h(\xi)+\xi}$ (по теореме Барроу $\psi$ есть непрерывно дифференцируемая функция), $C_{1}=\exp \tilde{C}_{1}$, а $\tilde{C}_{1}-$ произвольная вещественная постоянная. Следовательно, дифференциальное уравнение (24) имеет первый интеграл $L \psi(\xi)=C_{1}$, или $\Psi(K, L)=C_{1}$, где $\Psi$ - линейно-однородная непрерывно дифференцируемая функция. Тогда общее решение дифференциального уравнения в частных производных первого порядка (24) имеет вид $\Lambda(K, L)=H(\Psi(K, L))$, где $H$ - произвольная непрерывно дифференцируемая функция, а $\Psi$ - линейно-однородная непрерывно дифференцируемая функция двух переменных $K$ и $L$.

На основании полученного аналитического представления функции $\Lambda$ и неотрицательности ПФ (1) получаем аналитическое представление (10).

Утверждение $\mathbf{1 1}^{\circ}$. По определению (11), на основании тождества (12), получаем, что ПФ (1) учитывает обобщенно нейтральный по Хиксу НТП вдоль линейных траекторий развития экономики (13), если и только если при некоторой функции $h$ имеет место тождество

$$
\frac{\partial_{L} F(K, L, t)}{\partial_{K} F(K, L, t)}=h(a K+b L)
$$

Необходимость. Пусть ПФ (1) учитывает НТП, обобщенно нейтральный по Хиксу вдоль линейных траекторий развития экономики (13). Докажем, что функцию (1) тогда можно представить в аналитической форме (14). Для этого решим линейное однородное дифференциальное уравнение в частных производных первого порядка

$$
\partial_{L} F-h(a K+b L) \partial_{K} F=0
$$

с характеристической системой

$$
\frac{d K}{-h(a K+b L)}=\frac{d L}{1}=\frac{d t}{0}
$$

Обыкновенное дифференциальное уравнение $\frac{d K}{d L}=-h(a K+b L)$, введя новую переменную $\xi=a K+b L$, перепишем в виде $d L=\frac{d \xi}{b-a h(\xi)}$. Отсюда получаем

$$
L+\int \frac{d \xi}{a h(\xi)-b}=C_{1}, \text { или } L+\Psi(a K+b L)=C_{1},
$$

где положено $\Psi(\xi)=\int \frac{d \xi}{a h(\xi)-b}$ (по теореме Барроу $\Psi$ есть непрерывно дифференцируемая функция), $C_{1}$ - произвольная вещественная постоянная. Следовательно, характеристическая система (27) для уравнения (26) имеет первый интеграл $L+\Psi(a K+b L)=C_{1}$. 
Из уравнения $\frac{d L}{1}=\frac{d t}{0}$ находим первый интеграл $t=C_{2}$ характеристической системы (27), где $C_{2}$ - произвольная вещественная постоянная.

Следовательно, общее решение уравнения в частных производных (26) имеет вид $F(K, L, t)=\Phi(L+\Psi(a K+b L), t)$, где $\Phi-$ произвольная непрерывно дифференцируемая функция двух переменных, а $\Psi$ - непрерывно дифференцируемая функция одного аргумента.

Таким образом, имеет место аналитическое представление (14).

Достаточность. Пусть ПФ (1.1) представима в форме (14). Тогда, используя формулу вычисления производной сложной функции, находим предельную норму замещения

$$
\begin{aligned}
\frac{\partial_{L} F(K, L, t)}{\partial_{K} F(K, L, t)}= & \frac{\partial_{L} \Phi(L+\Psi(a K+b L), t)}{\partial_{K} \Phi(L+\Psi(a K+b L), t)}=\frac{\partial_{\xi} \Phi(\xi, t)_{\mid \xi=L+\Psi(a K+b L)} \partial_{L}(L+\Psi(a K+b L))}{\partial_{\xi} \Phi(\xi, t)_{\mid \xi=L+\Psi(a K+b L)} \partial_{K}(L+\Psi(a K+b L))}= \\
& =\frac{1+\partial_{\zeta} \Psi(\zeta)_{\mid \zeta=a K+b L} \partial_{L}(a K+b L)}{\partial_{\zeta} \Psi(\zeta)_{\mid \zeta=a K+b L} \partial_{K}(a K+b L)}=\frac{1+b \partial_{\zeta} \Psi(\zeta)_{\mid \zeta=a K+b L}}{a \partial_{\zeta} \Psi(\zeta)_{\mid \zeta=a K+b L}},
\end{aligned}
$$

а значит, при $h(\zeta)=\left(1+b \partial_{\zeta} \Psi(\zeta)\right) /\left(a \partial_{\zeta} \Psi(\zeta)\right)$ верно тождество (25).

Следовательно, динамическая ПФ (1) учитывает НТП, обобщенно нейтральный по Хиксу вдоль линейных траекторий развития экономики (13).

Утверждение $12^{\circ}$. По утверждению $11^{\circ}$, ПФ (1) учитывает обобщенно нейтральный по Хиксу НТП вдоль траекторий развития экономики (13) тогда и только тогда, когда ее можно представить в виде (14). Из условия однородности степени $q$ ПФ (1), используя аналитическое представление (14), получаем, что $\quad F(\lambda K, \lambda L, t)=\Phi(\lambda L+\Psi(\lambda(a K+b L)), t), \quad \forall \lambda \in(0 ;+\infty) \quad$ и $\lambda^{q} F(K, L, t)=\lambda^{q} \Phi(L+\Psi(a K+b L), t), \quad \forall \lambda \in(0 ;+\infty)$, а значит, функция $\Psi$ является однородной степени $q$ по переменной $L+\Psi$, а функция $\Psi$ является линейно-однородной по переменной $a K+b L$. Поэтому имеет место аналитическое представление

$$
\begin{gathered}
F(K, L, t)=\Phi(L+\Psi(a K+b L), t)=(L+\Psi(a K+b L))^{q} \Phi(1, t)= \\
=A(t)(L+(a K+b L) \Psi(1))^{q}=A(t)\left(a_{1} K+b_{1} L\right)^{q},
\end{gathered}
$$

где положено, что $A(t)=\Phi(1, t)$, вещественные числа $a_{1}=a \Psi(1), b_{1}=b \Psi(1)+1$.

Таким образом, верно аналитическое преставление (15).

Утверждение 15 $^{\circ}$ Если ПФ (1) учитывает НТП, нейтральный по Харроду, то, на основании утверждения $13^{\circ}$, ее можно представить в форме (16). Тогда

$$
\frac{\partial_{t} F(K, L, t)}{\partial_{L} F(K, L, t)}=\frac{\partial_{t} \Phi(K, \Psi(L, t))}{\partial_{L} \Phi(K, \Psi(L, t))}=\frac{\partial_{\zeta} \Phi(K, \zeta)_{\mid \zeta=\Psi(L, t)} \partial_{t} \Psi(L, t)}{\partial_{\zeta} \Phi(K, \zeta)_{\mid \zeta=\Psi(L, t)} \partial_{L} \Psi(L, t)}=\Theta(L, t)
$$

т. е. верно тождество (17) при $\Theta(L, t)=\partial_{t} \Psi(L, t) / \partial_{L} \Psi(L, t)$.

Утверждение $\mathbf{1 7}^{\circ}$. Однородная степени $q \in \mathbf{R} \backslash\{0\}$ ПФ (1) учитывает нейтральный по Хиксу НТП тогда и только тогда, когда она может быть представлена в виде (утверждение $2^{\circ}$ )

$$
F(K, L, t)=\tilde{A}(t) \tilde{F}(K, L),
$$


где $\tilde{F}$ - некоторая неотрицательная однородная степени $q$ непрерывно дифференцируемая функция. Если, кроме того, динамическая ПФ (1) учитывает нейтральный по Харроду НТП, то для функции (28) имеет место тождество (17), т. е. $\frac{\tilde{A}^{\prime}(t)}{\tilde{A}(t)} \cdot \frac{\tilde{F}(K, L)}{\partial_{L} \tilde{F}(K, L)}=\Theta(L, t)$, или

$$
\frac{\tilde{F}(K, L)}{\partial_{L} \tilde{F}(K, L)}=\frac{\tilde{A}(t)}{\tilde{A}^{\prime}(t)} \cdot \Theta(L, t) .
$$

Так как функция $\tilde{F}$ является однородной степени $q$, то функция $\partial_{L} \tilde{F}$ будет однородной степени $q-1$. Поэтому функция $f(K, L)=\tilde{F}(K, L) / \partial_{L} \tilde{F}(K, L)$, а значит, на основании тождества (29), и функция $\Theta(L, t)$ будет линейно-однородной по переменным $K$ и $L$. Следовательно, функция $\Theta(L, t)=L \cdot \Theta(1, t)$ и тождество (29) примет вид

$$
\frac{\tilde{F}(K, L)}{L \cdot \partial_{L} \tilde{F}(K, L)}=\frac{\tilde{A}(t)}{\tilde{A}^{\prime}(t)} \cdot \Theta(1, t)
$$

Это тождество имеет место, если и только если $\frac{\tilde{A}(t)}{\tilde{A}^{\prime}(t)} \cdot \Theta(1, t)=\frac{1}{\beta}, \beta \in \mathbf{R} \backslash\{0\}$.

Таким образом, функция $\tilde{F}$ есть решение дифференциального уравнения в частных производных первого порядка $\frac{1}{\beta} L \partial_{L} \tilde{F}=\tilde{F}$. Из характеристического уравнения $\frac{\beta d L}{L}=\frac{d \tilde{F}}{\tilde{F}}$ находим, что $\ln \tilde{F}-\beta \ln L=\tilde{C}(K)$, или $\tilde{F}(K, L)=L^{\beta} C(K)$, где $C(K)=\exp \tilde{C}(K)$, а $\tilde{C}(K)-$ произвольная функция (постоянная интегрирования). Отсюда, с учетом однородности степени $q$ функции $\tilde{F}$, получаем представление $\tilde{F}(K, L)=a K^{q-\beta} L^{\beta}$, число $a>0$. Полагая $A(t)=a \tilde{A}(t)$ и $\alpha=q-\beta$, получаем, что функция (28) имеет форму Викселля - Тинбергена (18).

Достаточность. Пусть динамическая ПФ имеет аналитическую форму (18). Тогда, по утверждению $2^{\circ}$, она учитывает НТП, нейтральный по Хиксу. А на основании утверждения $13^{\circ}$ и с учетом того, что функцию (18) можно представить в форме (16) с внешней линейно-однородной функцией $\Phi(K, \Psi)=K^{\alpha} \Psi^{1-\alpha}$ и внутренней функцией $\Psi(L, t)=\left(A(t) L^{\beta}\right)^{1 /(1-\alpha)}$, заключаем, что функция (18) учитывает НТП, нейтральный по Харроду.

Отметим, что утверждение $17^{\circ}$ для случая линейно-однородной динамической ПФ $(\alpha+\beta=1)$ было доказано Х. Удзава в работе (Uzawa, 1961).

Утверждение $19^{\circ}$. По определению (19) получаем, что функция (1) будет учитывать обобщенно нейтральный по Харроду НТП, если и только если имеет место зависимость между предельной производительностью капитала и средней отдачей обобщенного ресурса (20).

Необходимость. Пусть ПФ (1) учитывает НТП, обобщенно нейтральный по Харроду. Докажем, что ПФ (1) можно представить в аналитическом виде (21). Для этого решим квазилинейное уравнение в частных производных

$$
\partial_{K} F=h\left(\frac{F}{\alpha K+\beta L}\right)
$$

с характеристической системой 


$$
\frac{d K}{1}=\frac{d L}{0}=\frac{d t}{0}=\frac{d F}{h(F /(\alpha K+\beta L))}
$$

где $h$ - непрерывно дифференцируемая функция одной переменной.

Из обыкновенных дифференциальных уравнений $\frac{d K}{1}=\frac{d L}{0}$ и $\frac{d K}{1}=\frac{d t}{0}$ находим два первых интеграла $L=C_{1}$ и $t=C_{2}$ характеристической системы (31), где $C_{1}$ и $C_{2}$ - произвольные неотрицательные постоянные.

Дифференциальное $\quad$ уравнение $\quad \frac{d K}{1}=\frac{d F}{h(F /(\alpha K+\beta L))}, \quad$ введя $\quad$ новую $\quad$ переменную $\xi=F /(\alpha K+\beta L)$ и учитывая, что $L=C_{1}$, запишем в виде $\frac{d K}{\alpha K+\beta C_{1}}+\frac{d \xi}{\alpha \xi-h(\xi)}=0$. Интегрируя это дифференциальное уравнение, имеем $\frac{1}{\alpha} \ln \left(\alpha K+\beta C_{1}\right)+\int \frac{d \xi}{\alpha \xi-h(\xi)}=\tilde{C}_{3}$, или, потенцируя тождество и учитывая сделанные замены, получаем $(\alpha K+\beta L) g\left(\frac{F}{\alpha K+\beta L}\right)=C_{3}$, где положено, что функция $g(\xi)=\exp \int \frac{\alpha d \xi}{\alpha \xi-h(\xi)}$, а $C_{3}=\exp \left(\alpha \tilde{C}_{3}\right)$ и $\tilde{C}_{3}-$ произвольные вещественные постоянные.

Будучи функционально независимыми, первые интегралы $L=C_{1}, \quad t=C_{2} \quad$ и $(\alpha K+\beta L) g\left(\frac{F}{\alpha K+\beta L}\right)=C_{3}$ образуют интегральный базис системы (31). Тогда соотношение

$$
V(L, t,(\alpha K+\beta L) g(F /(\alpha K+\beta L)))=0,
$$

где $V$ - произвольная дифференцируемая функция трех аргументов, задает в неявном виде решение квазилинейного уравнения (30). При этом на основании теоремы 3.1 из работы (Егоров, 2007. C. 340-341) заключаем, что соотношение (32) определяет общее решение квазилинейного уравнения (30). Разрешая (32) относительно третьего аргумента, находим

$$
(\alpha K+\beta L) g\left(\frac{F}{\alpha K+\beta L}\right)=\Psi(L, t), \text { или } g\left(\frac{F}{\alpha K+\beta L}\right)=\frac{\Psi(L, t)}{\alpha K+\beta L},
$$

где $\Psi$ - произвольная непрерывно дифференцируемая функция двух переменных $L$ и $t$. А по теореме существования обратной функции ${ }^{28}$ из последнего тождества имеем $\frac{F}{\alpha K+\beta L}=g^{-1}\left(\frac{\Psi(L, t)}{\alpha K+\beta L}\right)=Q\left(\frac{\Psi(L, t)}{\alpha K+\beta L}\right)$, где $g^{-1}$ есть обратная к $g$ функция, а $Q-$ произвольная функция. На основании этого представления получаем, что решением квазилинейного дифференциального уравнения (30) будет функция

$$
F(K, L, t)=(\alpha K+\beta L) Q\left(\frac{\Psi(L, t)}{\alpha K+\beta L}\right)=(\alpha K+\beta L) \Phi\left(1, \frac{\Psi(L, t)}{\alpha K+\beta L}\right)=\Phi(\alpha K+\beta L, \Psi(L, t)),
$$

28 Фихтенгольц Г.М. 2001. Основы математического анализа: учебник. СПб.: Лань. Т. 1. С. 132-133. 
где $\Phi$ - произвольная линейно-однородная неотрицательная непрерывно дифференцируемая функция, а $\Psi$ - произвольная непрерывно дифференцируемая функция.

Достаточность. Пусть для ПФ (1) имеет место представление (21). Тогда, повторяя проведенные выше вычисления в обратном порядке, получаем, что (21) является решением уравнения (30), а значит, будет учитывать НТП, обобщенно нейтральный по Харроду.

Утверждения $20^{\circ}, \mathbf{2 9}^{\circ}$ относительно однородных ПФ доказываются аналогично утверждениям $2^{\circ}$ и $12^{\circ}$, а Утверждение $28^{\circ}$ получено методом, аналогичным использованному при доказательстве Утверждения $1^{\circ}$.

Полученные в работе результаты могут быть использованы при моделировании реальных производственных процессов, учитывающих НТП.

\section{СПИСОК ЛИТЕРАТУРЫ (REFERENCES)}

Анчишкин А.И. 2003. Прогнозирование темпов и факторов экономического роста. Москва: MAKC Преcc. [Anchishkin A.I. 2003. Forecasting economic growth rates and factors. Moscow: MAKS Press. (In Russ.)]

Барро Р.Дж., Сала-и-Мартин Х. 2017. Экономический рост. Москва: БИНОМ. [Barro R.J., Sala-i-Martin X. 2017. Economic growth. Moscow: BINOM. (In Russ.)]

Браун М. 1971. Теория и измерение технического прогресса. Москва: Статистика. [Brown M. 1971. On the theory and measurement of technological change. Moscow: Statistika. (In Russ.)]

Варшавский A.E. 1984. Научно-технический прогресс в моделях экономического развития: методы анализа и оценки. Москва: Финансы и статистика. [Varshavskiy A.E. 1984. Scientific and technical progress in models of economic development: methods of analysis and evaluation. Moscow: Finansy i statistika. (In Russ.)]

Гамецкий А.Ф., Соломон Д.И. 1997. Математическое моделирование макроэкономических процессов. Кишинэу: Еврика. [Gametskiy A.F., Solomon D.I. 1997. Mathematical modeling of macroeconomic processes. Kishineu: Evrika. (In Russ.)]

Егоров А.И. 2007. Обыкновенные дифференииальные уравнения с приложениями. Москва: Физматлит. [Egorov A.I. 2007. Ordinary differential equations with applications. Moscow: Fizmatlit. (In Russ.)]

Клейнер Г.Б. 1986. Производственные функиии: теория, методы, применение. Москва: Финансы и статистика. [Kleyner G.B. Production functions: theory, methods, application. Moscow: Finansy i statistika. (In Russ.)]

Курзенев В., Матвеенко В. 2018. Экономический рост. СПб.: Питер. [Kurzenev V., Matveenko V. 2018. Economic growth. Saint-Petersburg: Piter. (In Russ.)]

Плакунов М.К., Раяцкас Р.Л. 1984. Производственные функиии в экономическом анализе.
Вильнюс: Минтис. [Plakunov M.K., Rayatskas R.L. 1984. Production functions in economic analysis. Vilnius: Mintis. (In Russ.)]

Хацкевич Г.А., Проневич А.Ф. 2017. Квазиоднородные производственные функции единичной эластичности замещения факторов по Хиксу. Экономика, моделирование, прогнозирование. Вып. 11. C. 135-140. [Khatskevich G.A., Pranevich A.F. 2017. Quasi-homogeneous production functions with unit elasticity of factors substitution by Hicks. Ekonomika, modelirovanie, prognozirovanie. Vol. 11. PP. 135-140. (In Russ.)]

Хацкевич Г.А., Проневич А.Ф., Чайковский М.В. 2019. Двухфакторные производственные функции с заданной предельной нормой замещения. Экономическая наука сегодня. Вып. 10. С. 171-182. [Khatskevich G.A., Pranevich A.F., Chaykovskiy M.V. 2019. Two-factor production functions with given marginal rate of substitution. Ekonomicheskaya nauka segodnya. Vol. 10. PP. 171-182. (In Russ.)]

Beckmann M.J., Sato R. 1969. Aggregate production functions and types of technical progress: a statistical analysis. The American Economic Review. Vol. 59. No 1. PP. 88-101.

Besomi D. 1999. Harrod on the classification of technological progress. The origin of a wild-goose chase. PSL Quarterly Review. Vol. 52. No 208. PP. 95-117.

Blackorby Ch., Lovell C.A.K., Thursby M.C. 1976. Extended Hicks neutral technical change. The Economic Journal. Vol. 35. No 344. PP. 845-852.

Chilosi A., Gomulka S. 1969. Technical progress and long-run growth. Rivista di Politica Economica. No 3. PP. 1-47.

Ferguson C.E. 1969. The neoclassical theory of production and distribution. London: Cambridge University Press.

Gehrig W. 1980. On certain concepts of neutral technical progress: definitions, implications and compatibility. The economics of technological progress: Proceedings of a conference held by the European Production Study Group in Umea, Sweden, London: THE MACMILLAN PRESS LTD. PP. 3-21.

Khatskevich G.A., Pranevich A.F. 2017. On quasi-homogeneous production functions with constant 
elasticity of factors substitution. Journal of Belarussian State University. Economics. No 1. PP. 46-50.

Khatskevich G.A., Pranevich A.F. 2018. Production functions with given elasticities of output and production. Journal of Belarussian State University. Economics. No 2. PP. 13-21.

Khatskevich G., Pranevich A., Karaleu Yu. 2019. Analytical forms of productions functions with given total elasticity of production. Advances in Intelligent Systems and Computing. Vol. 1052. PP. 276-285.

Leontief W. 1947. Introduction to a theory of the internal structure of functional relationships. Econometrica. Vol. 15. No 4. PP. 361-373.

Morimoto Y. 1974. Neutral technical progress and the separability of the production function. The Economic Studies Quarterly. Vol. 25. No 3. PP. 66-69.

Okuguchi K. 1968. A note on Harrod neutral technical progress. Metroeconomica. Vol. 20. No 1. PP. 50-54.

Osiatynski J. (Ed.) 1993. Collected works of Michal Kalecki: Volume IV: Socialism: Economic growth and efficiency of investment. Oxford: Clarendon Press.

Robinson J. 1938. The classification of inventions. The Review of Economic Studies. Vol. 5(2). PP. 139-142.
Sato K. 1965. A note on the classification of technical inventions. The Economic Studies Quarterly. Vol. 15(2). PP. 73-77.

Sato R., Beckmann M.J. 1968. Neutral inventions and production functions. The Review of Economic Studies. Vol. 35(1). PP. 57-67.

Solow R.M. 1960. Investment and technical progress. Mathematical methods in the social sciences. Stanford: Stanford University Press. PP. 89-104.

Solow R.M. 1962. Technical progress, capital formation, and economic growth. The American Economic Review. Vol. 52(2). PP. 76-86.

Solow R.M. 1963. Capital theory and the rate of return. Amsterdam: North-Holland Publ. Comp.

Sono M. 1961. The effect of price changes on the demand and supply of separable goods. International Economic Review. Vol. 2. No 3. PP. 239-271.

Stiglitz J.E., Uzawa H. 1969. Readings in the modern theory of economic growth. Cambridge (Massachusetts): MIT Press.

Tinbergen J. 1942. Professor Douglas' Production Function. Review of the International Statistical Institute. Vol. 10. No 1/2. PP. 37-48.

Uzawa H. 1961. Neutral inventions and the stability of growth equilibrium. The Review of Economic Studies. Vol. 28(2). PP. 117-124.

In citation: Belorusskiy Economicheskiy zhurnal. 2020. No 3. PP. 87-105.

Belarusian Economic Journal. 2020. No 3. PP. 87-105.

\title{
TECHNOLOGICAL PROGRESS AND NEUTRALITY BY HICKS, HARROD, AND SOLOW: GENESIS, CONSTRUCTION, AND GENERALIZATIONS
}

\author{
Andrei Pranevich ${ }^{1}$, Guennadi Khatskevich ${ }^{2}$ \\ Authors affiliation: ${ }^{1}$ Yanka Kupala State University of Grodno (Grodno, Belarus); \\ ${ }^{2}$ School of Business of Belarusian State University (Minsk, Belarus). \\ Corresponding author: Andrei Pranevich (pranevich@grsu.by).
}

ABSTRACT. The article analyzes concepts of technological progress neutrality according to Hicks, Harrod, and Solow. It also describes sets of aggregate production functions inclusive neutral technological progress, extended Hicks neutral technological progress and complete Hicks neutral technological progress. Besides, analytical types of dynamic production functions are specified with the view of Harrod neutral technological progress and Solow neutral technological progress. Additionally we designate classes of homogeneous production functions responsive to Hicks neutral technological progress, Harrod neutral technological progress and Solow neutral technological progress. Analytical links between such concepts as neutral, extended neutral and complete Hicks neutral technological progress are revealed. All issues covered the article suggests generalizations of Hicks-neutrality, Harrod-neutrality, Solow neutrality concepts and correlates them with the corresponding sets of aggregate dynamic production functions. Analytical criteria that aggregate production functions have Hicks neutral technological progress, Harrod neutral technological progress and Solow neutral technological progress are given. The results obtained in this article can be used for modeling real production processes as part of technological progress.

KEYWORDS: technological progress, production function, Hicks neutrality, Harrod neutrality, Solow-neutrality.

JEL-code: C65, O30.

DOI: $10.46782 / 1818-4510-2020-3-87-105$

Received 2.07.2020 